\title{
Comparison of computational and semi-empirical aerodynamics tools for making fit-for-purpose modelling decisions
}

\author{
$\underline{\text { Dineth Abeynayake }}^{\text {a }}$, Alan Agon ${ }^{\text {a }}$ \\ ${ }^{a}$ Weapons Systems Division, Defence Science and Technology Organisation, South Australia \\ Email: dineth.abeynayake@dsto.defence.go.au
}

The weapons modelling and simulation community relies on a suite of aerodynamic data generation tools that span the empirical to numerical solution methods. These tools compute aerodynamic coefficients at differing fidelities and computational costs, where the tools that require the least amount of computational cost are also usually the least accurate. The intended result of this study was to define a set of best practices for frequently used aerodynamics packages. Missile DATCOM, NASA Cart3D and Metacomp CFD++ were chosen as a representative subset of the existing aerodynamics tools. This study investigated two distinct missile systems spanning the subsonic, transonic and supersonic flight regimes. Generated lift, drag and pitching moment coefficients from the three software packages were compared against wind tunnel data.

It was found that CFD++ produced the lowest errors of all the tools tested, highlighting the applicability of the tool to all tested flow regimes, and is recommended for use where the highest fidelity solution is desired and the result is not highly time critical. The results for Cart3D showed that it may be used to obtain a solution for supersonic cases where a small margin for error in the results is acceptable. However, the accuracy was lower for other flow regimes, especially the transonic regime where Cart3D produced erroneous results. Missile Datcom produced erroneous results for the non-conventional geometry tested, however it was able to produce medium fidelity results for conventional air to air missile shapes. The ability of Missile Datcom to produce results very quickly highlights its utility for quick-look analysis of conventional air to air systems.

The quantified errors and solution times are presented in a format that will aid modellers in making appropriate fit-for-purpose decisions for aerodynamic data generation as well as in assisting managers in deciding project milestones and delivery dates.

Keywords: $\quad C F D++$, Missile Datcom, Cart3D, validation, wind tunnel, comparison 


\section{INTRODUCTION}

The aerodynamic tools in this particular study have not been benchmarked against wind tunnel data within the weapons modelling and simulation community, thus there is ambiguity in the applicability of the different aerodynamic tools when analysing weapons systems. This comparison study aimed to determine which aerodynamic tools were most appropriate for a given scenario given the type of system under investigation as well as the operating environment. This study focussed on the three main tools used when generating aerodynamic data for a weapon system model, namely Missile Datcom, Cart3D and CFD++ and compared them with reference wind tunnel data. Two distinct, existing weapons systems were selected for the validation study. System A is an air to air missile, comprised of simple geometrical shapes including an ogive nose, cylindrical body and regular control surfaces, allowing it to be used as a benchmark for standard air to air systems. System B is a subsonic cruise missile and was used to determine which aerodynamic tools could predict accurate results for more complex non-conventional designs.

Missile Datcom, although primarily designed to be used as a preliminary aerodynamics design tool for missile shapes composed using basic geometric shapes such as cones, cylinders, etc, has become an analysis tool used quite extensively for weapons modelling and simulation. Datcom aims to provide very quick aerodynamic data sets including all static and dynamic aerodynamic derivatives using a combination of empirical and analytical methods (Missile Datcom User's Manual, 2008), and can produce solutions in under an hour including geometry and case setup costs. The relative ease of use has become a double edged sword as users with non-aerodynamics backgrounds may find themselves generating erroneous results with little to no warning about their fidelity.

Cart3D is a Cartesian-grid Euler solver with a fast solution turn-around time, and is typically used to perform the aerodynamic analysis of external geometry. Unlike Missile Datcom, Cart3D cannot generate input geometry and a CAD model must be externally created and imported into the solver. Cart3D is a mid-fidelity CFD solver where the initial geometry and case setup cost is in the order of days to weeks. Once an analysis is set up, cases can be solved in under an hour. Cart3D solves for the steady-state flow solution faster than $\mathrm{CFD}++$, as it does not model the viscous terms in the flow. Another feature of Cart3D is automated mesh adaption where the solver refines the mesh as a flow solution is being calculated based on outputs from a user specified fitness function applied over the flow field (Nemec et al, 2007).

$\mathrm{CFD}++$ is a high-fidelity Navier-Stokes solver with ability to model complex phenomena such as combustion and reacting flows (CFD++ User Manual, 2009). It has a large turn around time due to the fact that it can simulate high-order phenomena within the flow such as eddies, boundary layers and the like. A solution grid must also be generated manually in an external program (MIME) using CAD input geometry. Generating a complete solution in CFD++ takes in the order of weeks to months, however additional cases can be run every couple of days once the initial mesh is generated and a valid solution method is chosen.

\section{SYSTEMS TESTED}

\subsection{System A - Conventional Missile}

System A was chosen as the available wind tunnel data covered the Mach range of 0.5 to 5.0. The system has an axis-symmetric body with conventional control fins and an ogive nose. Supersonic cases at Mach 4.5 and transonic cases at Mach 0.95 at pitch angles of $0^{\circ}, 5^{\circ}$ and $10^{\circ}$ were run in each of the tested aerodynamic tools. These results were compared with wind tunnel data obtained for sea level free-stream conditions. The CAD geometry of this system is shown in Figure 1 below.

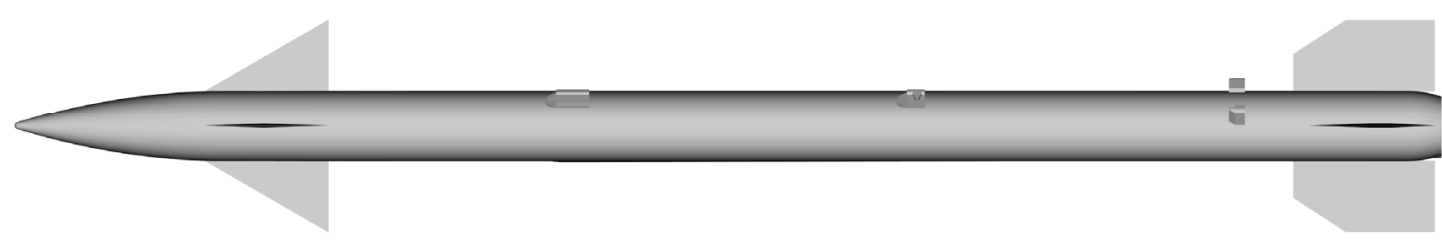

Figure 1. System A CAD Geometry 


\subsection{System B - Non-conventional Missile}

System B features a more complex missile geometry and hence a relatively complex fluid flow about the vehicle. System B baseline wind tunnel data was obtained for the cruise configuration with wings deployed. It is particularly important to compare how Missile Datcom handles such systems as it is based on empirical data from conventional missile geometries.

Only a single Mach number for System B was investigated where the missile was flying at a cruise speed of Mach 0.8 over the angle of attack range of $-10^{\circ}$ to $+10^{\circ}$ in $5^{\circ}$ increments. The System B CAD geometry in its analysed configuration is shown in Figure 2 below. While no mass flow through the engine is modelled, the stagnation of air in the initial portion of the inlet was captured.

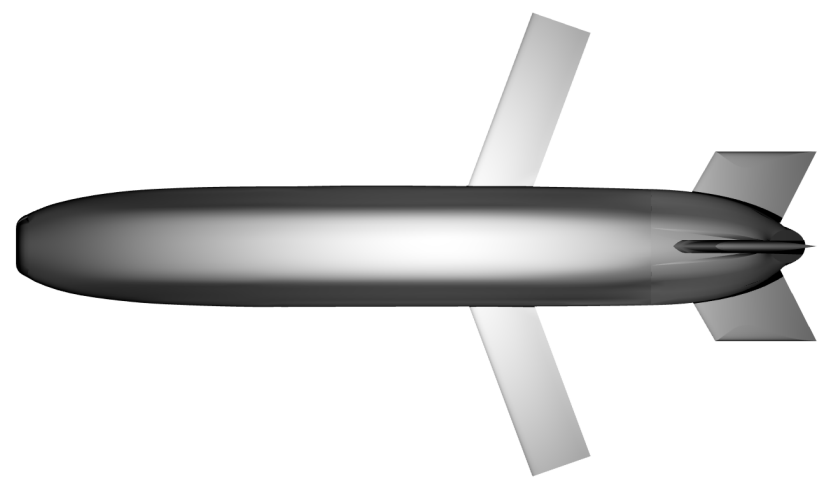

Figure 2. System B CAD Geometry

\section{RESULTS}

The three aerodynamic coefficients chosen for benchmarking were lift, drag and pitching moment due to their first order effects on axial and normal missile response when constructing simulation models. The relative errors across all three aerodynamic coefficients and all three solution methods have been tabulated in the discussion section. The experimental curves and the absolute values of the y-axis are not shown due to classification limitations.

\subsection{System A - Conventional Missile}

The drag results for the System A are shown for the transonic and supersonic cases in Figures 3 and 4 below. CFD++ most closely matched the System A wind tunnel drag results in both supersonic and transonic regimes. Missile Datcom performed well for both flow speeds and slightly over predicted drag while maintaining the correct trend when angle of attack was increased. As expected, Cart3D under-predicted drag however maintained the correct trend for supersonic flow speeds; although at transonic speeds the determined trend was incorrect. This is likely due to the inability of the solver to capture viscous effects such as skin friction (Anderson, 2006). The Cart3D drag values diverged from other results at higher angles of attack due to the flow becoming more susceptible to turbulent effects such as recirculation.

The lift results for System A obtained from the three tools are shown in Figure 6 and Figure 7 for transonic and supersonic cases respectively. CFD++ also provided the best match for lift coefficient for all tested flow speeds. Missile Datcom matched closely in the transonic regime however slightly over predicted and diverged as angle of attack was increased in the supersonic regime. Cart3D had

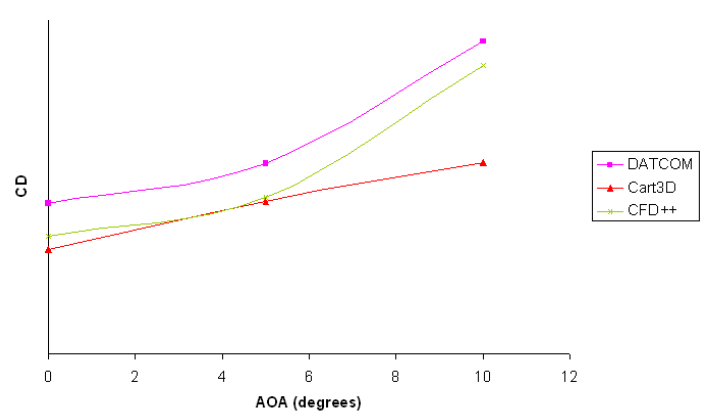

Figure 3. Drag coefficient System A Mach 0.95

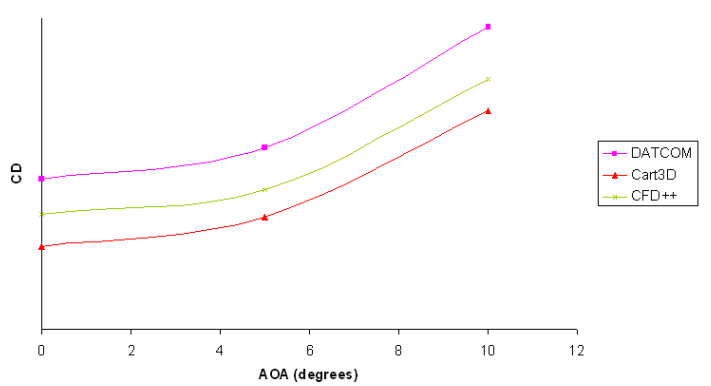

Figure 4. Drag coefficient System A Mach 4.5 
the most difference in accuracy of results when comparing between supersonic and transonic flow. In the supersonic regime Cart3D correlated well with wind tunnel results for all angles of attack tested, however at transonic speeds the slope of life curve was quite different to what was expected. As previously mentioned, wind tunnel results cannot be shown due to classification restrictions.

When comparing at transonic flow conditions the $\mathrm{CFD}++$ and Datcom results for pitching moment corresponded closely to wind tunnel results, however Cart3D displayed an incorrect moment value at zero angle of attack as well as an incorrect slope. All of the tools correctly estimated the slope for the pitching moment curve in the Mach 4.5 regime. CFD++ matched the wind tunnel data most closely. Cart3D estimated the supersonic pitch moments better than in the transonic regime; however Datcom was less accurate in its prediction for supersonic speeds.

\subsection{System B - Non-conventional Missile}

A comparison of the obtained drag and lift results for System B is shown in Figure 5 and Figure 8 respectively. CFD++ performed best compared to wind-tunnel results, followed by Cart3D and lastly by Missile Datcom. The results for Cart3D and Missile Datcom diverged at higher angles of attack indicating that the wake region aft of the body had not been captured correctly. All sources of data showed the asymmetry in drag forces due to the asymmetry of the vehicle in the pitch plane.

As expected, lift was better predicted by all of the aerodynamics packages. CFD++ was consistently closer to the experimental results than all of the other tools. It was able to match with experimental data very well with the exception of $0^{\circ}$ and $-5^{\circ}$ angle of attack. Although Cart3D matched well at the zero incidence $\alpha$ condition, the slope of the lift curve was poorly predicted when compared to all other sources. Missile Datcom was the only solver which predicted positive lift at zero $\alpha$, most likely due to the axis-symmetric approximations made for the aft section of the vehicle. Datcom lift results had a constant offset for lift, with approximately the correct slope.

The pitching moment had the largest variation in values and was evidently the most difficult to model. For lift and drag, CFD++ was closest to the baseline experimental data; however Cart3D matched the pitching moment results most closely. The trend observed for the Cart3D results did not correlate with the experimental results in this case. Each of the aerodynamics packages predicted different trends and angles where the vehicle was stable, leading to a large uncertainty within the pitching moment dataset, which have not been included for brevity.

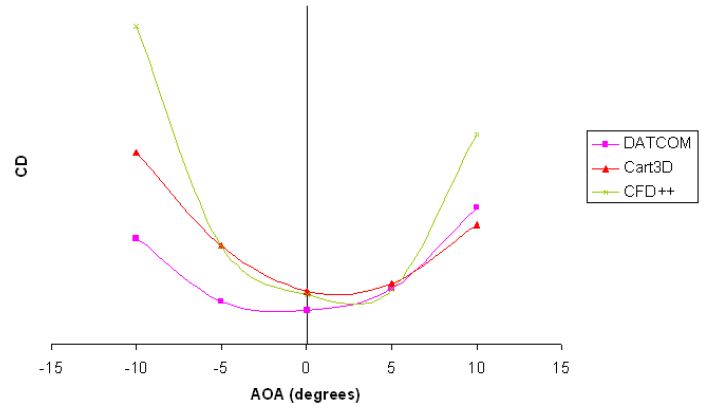

Figure 5. Drag coefficient System B Mach 0.8

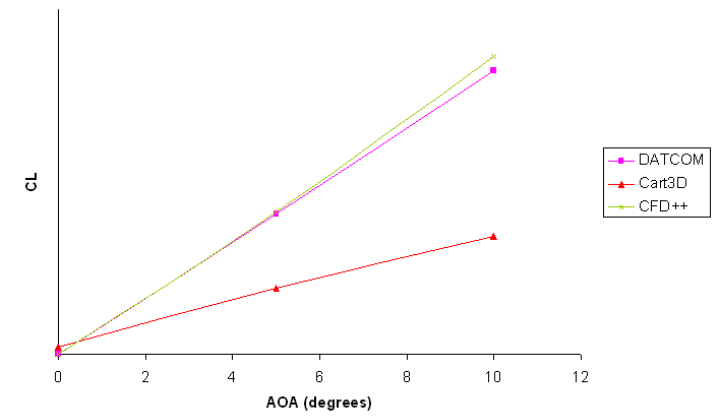

Figure 6. Lift coefficient System A Mach 0.95

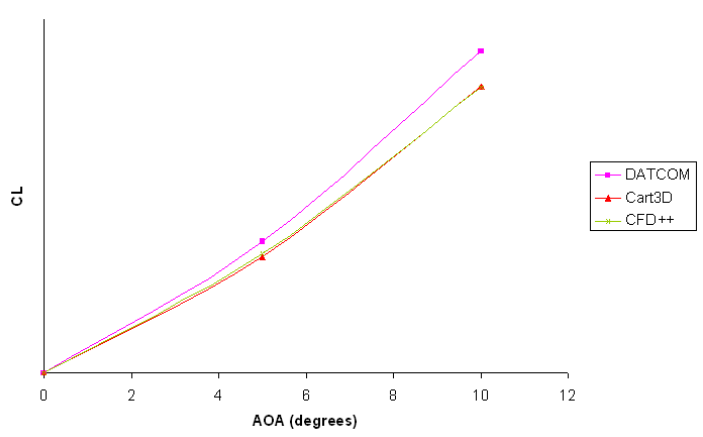

Figure 7. Lift coefficient System A Mach 4.5

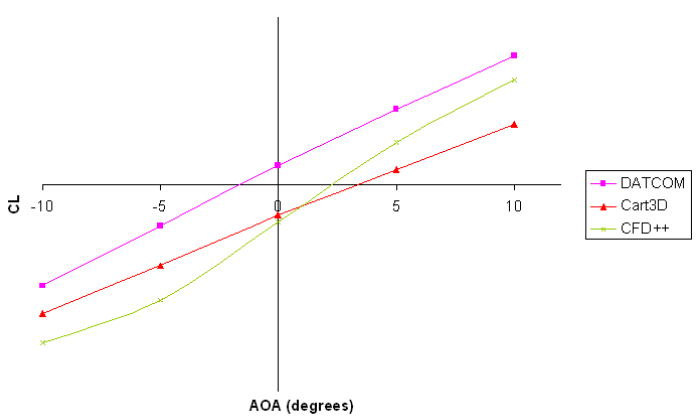

Figure 8. Lift coefficient System B Mach 0.8 


\section{DISCUSSION}

\subsection{Solution Time Comparison}

Table 1. Approximate regions of applicability of tested aerodynamic codes

\begin{tabular}{|c|c|c|c|c|}
\hline \multirow{2}{*}{ Aero Tool } & \multirow{2}{*}{ Solution Time } & \multicolumn{3}{|c|}{ Averaged Relative Error for all Tested Coefficients } \\
\cline { 3 - 5 } & & Conventional Air to Air Missile & Unconventional streamlined Missile \\
\cline { 3 - 5 } & & Transonic & Supersonic & Subsonic \\
\hline Datcom & $<1$ day & $25 \%$ & $22 \%$ & $36 \%$ \\
\hline Cart3D & $>3$ weeks & $38 \%$ & $15 \%$ & $23 \%$ \\
\hline CFD++ & $>1$ month & $4 \%$ & $5 \%$ & $6 \%$ \\
\hline
\end{tabular}

The total set-up times are indicative of a user with an aerodynamics background and a sound understanding of the software. The Cart3D solution times are based on a Intel Xeon 16 core CPU with 6GB of RAM. The $\mathrm{CFD}++$ cases are run across three Intel Xeon 16 core servers, each with $28 \mathrm{~GB}$ of RAM.

As seen in Table 1, Missile Datcom requires the least amount of solution time compared to the other tested software, as it is easy to use from creation of geometry to generation and visualisation of results. However, if the operator has not previously used the software, a period in the order of weeks is required for training to be able to produce initial results. This amount of time will be considerably less than the other programs. Once results are produced, it is important to conduct a sanity check to determine if the aerodynamic trends appear as expected - a process which should be undertaken by an aerodynamicist.

Datcom has a geometry library which can be used to specify the required system in a user friendly manner and can usually be completed within two hours. Assuming geometry is defined, flight conditions and other parameters are setup, the solution time is almost instantaneous. Once a result is obtained, iterations in the solution are usually not required. Multiple data points can be run simultaneously, therefore the total solution time is independent of number of data points. This makes the program suitable for generating large datasets.

Compared to Missile Datcom, Cart3D requires more time to arrive at a finalised solution. There is a high learning overhead to understand the adaptive meshing and solution process, even for operators with aerodynamics backgrounds. A sound knowledge of the program as well as an understanding of aerodynamics is required to generate correct results.

Geometry must be generated in CAD software and input into the Cart3D solver. The time required for this is a function of the complexity of the geometry and can take from a few days up to two weeks. Solution iterations will most likely be required to arrive at a valid mesh adaption strategy (Nemec et al, 2008), and this process can take up to 3 weeks, as seen in Table 1 . However, once a correct strategy is in place, running each data point takes approximately 2 hours. The total solution time will clearly be a function of how many data points are required and the hardware.

CFD++ has a very high learning curve due to high fidelity of software which requires a wide range of inputs from turbulence models to boundary conditions. The training process involved for both the meshing program and solver is in the order of several months. As a result, CFD++ requires the largest solution time out of all software packages tested, as shown in Table 1. An understanding of a separate meshing program lengthens the training time when compared to Cart3D as a human in the loop is required. Cart3D and CFD ++ , although having some similarities, are different to operate. As a consequence an operator who is able to use one of the programs will not necessarily be able to use the other without additional training.

The geometry creation process required is similar to Cart3D where CAD geometry must be imported into the meshing program. The mesh requires an initial solution to be generated before a visual inspection can be undertaken to ensure all flow features are resolved. Therefore multiple iterations may be required, each of which can take up to a week. The solution time for each data point can take up to 48 hours depending on the number of mesh elements.

\subsection{Result Comparison}

The relative error of each tool compared against wind tunnel data is presented for the three main coefficients of interest below. It is important to note that experimental data points within each system were chosen to 
non-dimensionalise the absolute error values. For example, an average of 0 and $10^{\circ}$ incidence $\alpha$ experimental data points were used to non-dimensionalise drag error for each system. However, to non-dimensionalise error in lift coefficient and pitch moment coefficient, the $10^{\circ} \alpha$ experimental data point was used due to the oscillation of the values about zero. Thus the relative errors may be used to compare one tool to another for a particular system, however these are only an indication of the exact accuracy of a specific tool.

Table 2. Drag Relative Error

\begin{tabular}{|c|c|c|c|c|c|c|}
\cline { 2 - 7 } \multicolumn{1}{c|}{} & \multicolumn{2}{c|}{ Datcom } & \multicolumn{2}{c|}{ Cart3D } & \multicolumn{2}{c|}{ CFD++ } \\
\hline CD & Mean & Max & Mean & Max & Mean & Max \\
\hline Subsonic & $47.4 \%$ & $142.7 \%$ & $31.3 \%$ & $80.2 \%$ & $6.8 \%$ & $11.0 \%$ \\
$\begin{array}{c}\text { (System B) } \\
\text { Transonic }\end{array}$ & $21.3 \%$ & $23.4 \%$ & $18.0 \%$ & $45.8 \%$ & $4.5 \%$ & $7.5 \%$ \\
(System A) & & & & & & \\
Supersonic & $17.5 \%$ & $34.9 \%$ & $23.5 \%$ & $25.3 \%$ & $6.4 \%$ & $12.7 \%$ \\
(System A) & & & & & & \\
\hline
\end{tabular}

It is clear from Table 2 that $\mathrm{CFD}++$ consistently determined drag values with the least relative error when compared to the other tools by a significant margin. While Cart3D provided relatively accurate results in the transonic regime when compared to subsonic, it was seen to be more precise in the supersonic regime. Cart3D also struggled to correctly resolve the wake region, which was more noticeable at higher incidence $\alpha$ angles, thus leading to incorrect drag- $\alpha$ slopes being predicted at lower speeds. Datcom provided reasonable drag values, however when dealing with non-standard missile shapes such as in the subsonic sonic cases tested, the errors were much larger.

Table 3. Lift Relative Error

\begin{tabular}{|c|c|c|c|c|c|c|}
\hline & \multicolumn{2}{|c|}{ Datcom } & \multicolumn{2}{|c|}{ Cart3D } & \multicolumn{2}{|c|}{ CFD++ } \\
\hline CL & Mean & Max & Mean & Max & Mean & Max \\
\hline $\begin{array}{c}\text { Subsonic } \\
\text { (System B) }\end{array}$ & $25.3 \%$ & $36.6 \%$ & $16.5 \%$ & $32.2 \%$ & $5.6 \%$ & $13.2 \%$ \\
\hline $\begin{array}{l}\text { Transonic } \\
\text { (System A) }\end{array}$ & $0.5 \%$ & $0.9 \%$ & $28.7 \%$ & $58.7 \%$ & $1.9 \%$ & $4.4 \%$ \\
\hline $\begin{array}{l}\text { Supersonic } \\
\text { (System A) }\end{array}$ & $7.5 \%$ & $15.8 \%$ & $1.3 \%$ & $3.2 \%$ & $1.6 \%$ & $2.9 \%$ \\
\hline
\end{tabular}

Table 3 shows the relative errors associated with lift, where $\mathrm{CFD}++$ provided the most accurate results followed by Cart3D and finally by Missile Datcom. This trend did not apply in the transonic regime where Datcom provided most accurate results. It should be noted that Datcom had a disadvantage in the System B lift data set as it was unable to predict body lift due to the axis-symmetric body libraries within it. However, the results indicated that Datcom may be used to produce lift values accurately when a quick look solution is sought for generic missile shapes. As expected, Cart3D was considerably less reliable in the transonic regime, where a stable solution was more difficult to obtain. CFD++ was again shown to outperform the other tools by a significant margin.

Table 4. Pitch Moment Relative Error

\begin{tabular}{|c|c|c|c|c|c|c|}
\cline { 2 - 7 } \multicolumn{1}{c|}{} & \multicolumn{2}{c|}{ Datcom } & \multicolumn{2}{c|}{ Cart3D } & \multicolumn{2}{c|}{ CFD++ } \\
\hline CM & Mean & Max & Mean & Max & Mean & \\
\hline Transonic & & & & $119.2 \%$ & $5.9 \%$ & $13.1 \%$ \\
(System A) & $3.2 \%$ & $5.1 \%$ & $67.1 \%$ & & & $9.4 \%$ \\
Supersonic & & & & $34.1 \%$ & $7.1 \%$ & \\
(System A) & $42.5 \%$ & $52.1 \%$ & $22.0 \%$ & & & \\
\hline
\end{tabular}


The pitch moment errors depicted in Table 4 were low for Datcom and CFD++ for transonic speeds, however Cart3D was less accurate in its prediction resulting in higher errors. For supersonic speeds Datcom's performance degraded, conversely Cart3D's improved while CFD++ maintained a roughly constant level of accuracy.

\section{CONCLUSION}

This study showed that Missile Datcom outperforms Cart3D when calculating aerodynamic lift, drag and pitching moment coefficients in the transonic regime for conventional airframe geometry. A Missile Datcom solution is based on empirical data which takes into account the large viscous effects present in any transonic flow. For such conventional missile geometries, Datcom was shown to be relatively accurate in its predictions and is suitable for preliminary analysis that needs to be conducted in an order of days. However, for less conventional missile geometries a higher fidelity tool must be used.

Cart3D was shown to provide aerodynamic parameters which were accurate for first order analysis, although only for supersonic speeds. For lower and especially transonic speeds Cart3D had trouble reaching stable solutions and under predicted drag. Cart3D was seen to be very useful for lift prediction in the speed regime of minimal errors, however where drag prediction is required it was not considerably more accurate than Datcom. Generating a correct Cart3D solution can take a number of weeks, and should be used where a higher fidelity solution than Datcom is required, especially for non-conventional geometries.

The CFD++ performed the best out of all the tested programs as results for the three parameters were within $7 \%$ of wind tunnel data for all tested flow conditions and geometries. CFD++ produced results more accurate than Missile Datcom with generic missile shapes and almost an order of magnitude more accurate in drag when comparing non-standard missile shapes such as System B. However, the time required to obtain a CFD++ solution is greater than the other lower fidelity programs, and a decision must be made if work at hand requires such a high fidelity solution before automatically deciding to use the software, especially where tight deadlines are in place.

Modelling and simulation fly-out model results are highly reliant on the inclusion of correct aerodynamic data. An error within these coefficients can greatly influence the requirements placed on other subsystems and modify the overall system performance. Observing all three variables, it can be generalised that the relative errors mirror the time overhead required to set the cases up. Results from this study can be used for calibrating each tested software in order for them to produce more accurate results, although is limited to the types of geometries and flow regimes tested. Great care should be taken when selecting the tool to generate the aerodynamics and the limitations of the package should be well understood.

\section{REFERENCES}

Anderson, JD. (2006). Hypersonic and High Temperature Gas Dynamics Second Edition, AIAA Education Series, Virginia

CFD++ User Manual (2009). Metacomp Technologies, USA

Missile Datcom User's Manual (2008). Air Force Research Laboratory, USA

Nemec, M. \& Aftosmis, M. (2007). 'Adjoint Error Estimation and Adaptive Refinement for EmbeddedBoundary Cartesian Meshes', in 18th AIAA Computational Fluid Dynamics Conference, AIAA, Miami, pp. 1-16

Nemec, M., Aftosmis, M. \& Wintzer, M. (2008). 'Adjoint-Based Adaptive Mesh Refinement for Complex Geometries', in 46th AIAA Aerospace Sciences Meeting, AIAA, Reno, pp. 1-23 